than those obtainable in this country. Dr. L. M. Orr, in the Journal of Urology, states that in six different American clinics the number of resections performed to one prostatectomy was as follows: $80,19,9,1.7,1.5,0.9$ respectively. To his question, On what type of case do you employ resection? circularized American surgeons returned answers that ranged from a frank denial that it was of any value to an assertion that it should be employed in every type of case. In England, perhaps owing to our happy genius for discovering middle courses, these extremes of opinion are seldom encountered. The majority of urologists have accepted perurethral resection as a valuable addition to our methods of treating the obstructing prostate, and use it in moderation. Each of us may differ in our interpretation of the word moderation, but our divergence is not great. Personally I mean by that word that I employ resection in about 20 per cent. of all my prostatic cases, reserving it in particular for minor enlargements or for patients in whom, for one reason or another, it would be dangerous to perform a prostatectomy. Because resection can be carried out in cases in which enucleation would be dangerous it appears to me to be the most valuable of recent additions to our methods of treatment. When combined with a temporary suprapubic cystotomy, done under local anaesthesia, it is by far the safest of all the surgical attacks on the obstructing prostate, and can be carried out on patients who formerly would have been condemned to catheter life or to a permanent suprapubic drainage. It provides, therefore, a welcome way out of many a difficult clinical problem.

In conclusion I should like to make a digression. For a long time I have felt that our methods of dealing with prostatic obstruction were in some danger of becoming over-standardized. There is a tendency for us to become champions of one or another particular brand of operation instead of exponents of them all. Personally $I$ am trying to avoid being pro-McCarthy, pro-Thomson, proHarris, pro-Freyer, or pro-Thomson-Walker, and employ each method where I believe it to be indicated. Each of these techniques has its place in our surgery; each its advantages and its shortcomings. In the preceding retrospect I have pointed out what in my opinion are the chief disadvantages of the McCarthy operation and have discussed some of the steps that urologists are now taking to remove them.

C. Weysser (Dtsch. med. Wschr., February 19, 1937, p. 307) remarks that some sixty different causes of abortion have been identified, but that it is very difficult to associate any one of them with a given case. The treatment of threatened abortion has till recently lacked specific direction, but now, with the recognition of the part played by vitamins and hormones in this matter, it is possible to be more rational and less empirical. The author gives an account of his experience with ninety-six cases of threatened abortion treated with the vitamin $D$ preparation vigantol. His rationale was based on the investigations of Poullson, who in 1924 drew attention to the favourable effect of cod-liver oil on intra-uterine development. The vigantol was administered three times a day, only one drop at a time. This treatment was continued for several weeks, and when possible to term. Other and older remedies for threatened abortion, such as opium and rest in bed for at least a week after the cessation of haemorrhage, were not neglected. This treatment was successful in about half the cases. The author chooses as controls the records of other gynaecologists whose patients were not treated with vitamin $D$ and whose results varied considerably but were always much inferior to those claimed for vitamin D medication.

\section{BLOOD TRANSFUSION IN OBSTETRICS}

BY

MALCOLM D. BLACK, M.B., Ch.B., F.R.F.P.S., M.C.O.G.

Lately Registrar to the Glasgow Royal Maternity and Women's Hospital

This article has been written to describe the formation of a blood transfusion service and to give an outline of the indications, method, and dangers of blood transfusion. Among the branches of medicine in which there is a place for blood transfusion obstetrics is pre-eminent; yet this fact has been neglected for many years on the ground that the pregnant or parturient woman stands blood loss better than any other type of patient.

As pointed out by Professor Dugald Baird in a lecture to the Glasgow Obstetrical Society and later published in the Lancet (1936), one of the outstanding needs of an efficient maternity hospital is a blood transfusion service. The great difficulty found by most institutions requiring large numbers of transfusions has been to secure a suitable donor promptly. In previous years patients have died after a stay of eight hours in this hospital, during which time twelve donors had been tested and found unsuitable. It is tirus desirable to have a list of grouped donors within easy call.

\section{Procedure in the Glasgow Royal Maternity and Women's Hospital}

In June, 1936, a service was started in this hospital. Placards had been posted at the entrance to it and in public places in the neighbourhood stating that male donors were urgently required, and that a fee of $£ 11 \mathrm{~s}$. would be paid to anyone giving blood. The prospective donors were asked to leave their names at the inquiry office. At a later date they were requested by postcard to come to the hospital for examination of the heart and lungs, Wassermann reaction, and blood group. If the heart and lungs were normal and the Wassermann reaction was negative the donor was placed on the roll and informed to that effect by another postcard. By this means a roll of $\mathbf{1 8 0}$ donors has gradually been built up. All groups are put on the roll.

Before a donor is sent for the recipient's blood is first grouped to determine the appropriate donor. The policy of the hospital, notwithstanding, is still to use a relative where possible. By this means a saving of universal donors is effected. However, in cases of great urgency a Group IV donor is summoned at the time of the patient's admission. When the occasion arises the motor hirers to the hospital are telephoned, given the names and addresses of three donors living in the same locality, and instructed to secure one donor as quickly as possible. In this way a donor can practically always be secured within thirty minutes.

When a donor arrives a rapid direct compatibility test is performed before the transfusion is started. The blood having been withdrawn the donor is kept lying flat for about one hour, given a hot meal, and then sent home by taxi. He also receives a form stating that he has given blood, and on production of this form at the house-superintendent's office he is paid the fee of $£ 11 \mathrm{~s}$. Should a donor be called and not used he is paid $5 \mathrm{~s}$. This service has been made available for the private practice of the members of the visiting staff of the hospital. Since the commencement of the service fifty donors have given blood and nineteen have been called and not used. By means of this service we hope to prevent as far as possible deaths from haemorrhage in the hospital. 


\section{Methods}

The test for compatibility is made either by nakedeye examination of a mixture of the donor's corpuscles with the recipient's serum, one drop of blood being added to two drops of serum, or by making a hanging-drop preparation of equal parts of a fine suspension of the donor's corpuscles, one drop of blood in $1 \mathrm{c.cm}$. of a 5 per cent. citrate solution, and the serum of the recipient. Agglutination will take place in five minutes if the bloods are incompatible. The test for agglutination makes unnecessary that for haemolysis, since agglutination always occurs before haemolysis. In transfusions performed outside the hospital a compatibility test can be performed after waiting about ten minutes for a little serum to separate off from the recipient's blood.

It may be noted here that in certain cases corpuscles from a universal donor, from a donor belonging to the same group as the patient, or from the patient herself have appeared on a cold slide to be incompatible with the recipient's serum. On warming the slide, however, the agglutination, which has never been absolutely definite, disappears. In all cases, wherever possible, two persons should make independent tests for compatibility.

In this hospital the indirect method of transfusion is always used. The blood is drawn off from the donor in a separate room by means of a French's venesection needle, to which is attached a few inches of rubber tubing. The blood is allowed to run into a flask containing $150 \mathrm{c.cm}$. of a 5 per cent. citrate solution. While the blood is being run into the solution a gentle rotatory movement is maintained to secure proper mixing in the flask. Thus $600 \mathrm{c.cm}$. of blood are drawn off, so that the flask contains $750 \mathrm{c.cm}$. fluid-namely, $600 \mathrm{c.cm}$. of blood and $150 \mathrm{c.cm}$. of 5 per cent. citrate solution, which gives a final 1 per cent. citrate mixture.

Instead of the ordinary tourniquet being applied to the donor's arm the arm-piece of a Baumanometer is used. By this method the constricting pressure can be maintained at about $60 \mathrm{~mm}$. $\mathrm{Hg}$. a figure well below the systolic blood pressure. It is not uncommon to find even in healthy young adults a sudden fall in blood pressure, probably of psychic origin, soon after the onset of the proceedings ; cutting down on to a donor's vein should never be permitted. It may be noted that the London blood transfusion service has wisely instructed their donors never to allow a surgeon to cut down on to the vein.

After the blood.has been secured a simple gravity saline infusion apparatus is used. Cutting down on to the recipient's vein is always advisable, as it is surer and quicker in collapsed patients. The vein is first ligatured distally, and a single loose knot is tied round the proximal end. The opening in the vein is best made transversely by a pair of sharp scissors, and the cannula is inserted, some saline being kept running through it during the process. When the cannula has been inserted the knot of the proximal ligature is drawn tight. If difficulty occurs in inserting the cannula the proximal flap of the incision in the vein may be lifted up with fine nontoothed dissecting forceps. This method is in our experience the simplest and quickest.

The Kimpton-Brown method with the flask and bellows has been discarded in this hospital on account of the difficulty in inserting the cannula into the vein and the liability of the cannula to slip out when the patient is restless. The usual amount given is 600 to $1,000 \mathrm{c.cm}$. An objection to the gravity method has been raised on account of the length of rubber tubing necessary, but in our experience no clotting or other difficulty has been found. Twenty minutes at least should be taken in introducing $600 \mathrm{c} . \mathrm{cm}$. of blood, otherwise an acute dilatation of the right side of the heart may be encountered. In cases of urgency, while the operator is securing blood the assistant can cut down upon the recipient's vein and so expedite the transfusion.

In very collapsed patients, such as cases of retained placenta with severe post-partum haemorrhage, it is occasionally almost impossible to keep the patient at rest while inserting a cannula or starting the blood transfusion. In these cases in our experience the best line of treatment is to give the patient a few drops of ether on an open mask. The patient becomes anaesthetized quickly and then lies at rest; coincidentally the general condition is much improved owing to the stimulant action of the ether. This procedure has been found to be much safer than an attempt to give a blood transfusion to a struggling patient who has to be forcibly controlled, especially since this state of restlessness is often a sign of impending death.

\section{Athrombit Method}

This non-citrate method has only been used on six occasions in this hospital. It is useful in cases of small blood transfusions, such as $300 \mathrm{c.cm}$. given to increase the patient's resistance, but it has been found unsuitable for the large transfusions to replace blood loss which are so often required in obstetrics.

\section{Transfusion Outside the Hospital}

In cases of extreme urgency it is often preferable to treat the patient by blood transfusion in her own home before transit to hospital. In these cases and in private practice generally the gravity method can be easily performed in the patient's home. It is better to draw off the blood in the hospital and carry it out to the patient, but in the case of patients living at a great distance from the hospital, or in private practice, the whole procedure may take place in the home.

\section{Indications}

The following table shows the various conditions treated by blood transfusion in the years 1935 and 1936 in the Glasgow Royal Maternity and Women's Hospital.

\begin{tabular}{c|c|c|c|c|c|c|c}
\hline & $\begin{array}{c}\text { Retained } \\
\text { Placenta } \\
\text { P.P.H. }\end{array}$ & $\begin{array}{c}\text { Placenta } \\
\text { Praevia }\end{array}$ & $\begin{array}{c}\text { Accidental } \\
\text { Haemor- } \\
\text { rhage }\end{array}$ & Abortion & $\begin{array}{c}\text { Obstetric } \\
\text { Shock and } \\
\text { Others }\end{array}$ & $\begin{array}{c}\text { Total } \\
\text { Cases }\end{array}$ & $\begin{array}{c}\text { Total } \\
\text { Trans- } \\
\text { fusion }\end{array}$ \\
\hline 1935 & 27 & 19 & 16 & 22 & 8 & 92 & 105 \\
1936 & 49 & 30 & 15 & 29 & 22 & 145 & 165 \\
\hline
\end{tabular}

The main object in obstetrics in giving blood transfusion has been to replace blood loss from haemorrhage. At times it has been given with a view to increasing the patient's resistance to infection.

\section{Very Collapsed Patients}

The question often arises whether it is too late to give a transfusion; while the patient is living it is not too late. To illustrate this point a case may be quoted of retained placenta and post-partum haemorrhage in which, just as the blood had been drawn off, the patient stopped breathing; the pulse could not be felt, and she was apparently dead. Intracardiac adrenaline injection, the administration of oxygen, and artificial respiration were started at once, and the blood transfusion was con- 
tinued. At the end of the first $900 \mathrm{c.cm}$. the patient was breathing; the pulse was just perceptible, but she was still unconscious. Since another donor was available more blood was secured; by the time $1,800 \mathrm{c.cm}$. had been given the patient was wide awake and conversing freely.

The question of giving intravenous saline solution to a patient requiring blood transfusion while waiting to secure blood requires discussion. If blood is not yet available and the patient is not at the point of death an intravenous saline injection should not be given; otherwise the blood pressure will be raised, the haemorrhage will recur, and she will soon be in a worse state than before. On the other hand, if blood will be available within half an hour to one hour, or if the patient be at the point of death, it is better to start giving an intravenous saline injection and to continue it until blood has been secured.

\section{Retained Placenta with Post-partum Haemorrhage}

It will be seen from the table that most cases requiring transfusion belong to this class. When the patient has received a transfusion the placenta must be removed by expression or manually; otherwise further bleeding will occur coincident with the rise of blood pressure. It is sometimes held that if the patient had previously been in a very collapsed state she should be left for some hours after transfusion before any operative procedure is undertaken. The delay often results in more bleeding, however. Many patients will lose nearly as much blood as they have been given, and require a further transfusion before operation.

If the patient at the end of the transfusion is still too weak to stand operative intervention more blood must be transfused till the patient is fit, at which time the operation should be undertaken. In some cases in the past when transfusion had been unsuccessful the amount of blood given was insufficient. Hence large quantities of blood are essential, at times up to $2,000 \mathrm{c.cm}$.

\section{Placenta Praevia}

Cases of placenta praevia very often require transfusion before operative procedures can be undertaken. Since these patients may lose a great deal of blood during the operation, preparation should be made to give a further transfusion in the course of it or immediately afterwards. As in cases of retained placenta all operative procedures should be undertaken soon after the tirst transfusion. A very important factor in the fall in the maternal mortality from-placenta praevia in the past few years has been the increased use of blood transfusion as an adjunct to operative treatment.

\section{Accidental Haemorrhage}

While very few people doubt the efficacy of blood transfusion in cases of placenta praevia, many hesitate to recommend it for cases of accidental haemorrhage. Examination of the mortality in cases of accidental haemorrhage reveals that blood loss is often an important factor. Transfusion is thus indicated in these cases. However, since there is often such a large element of shock, independently of the actual blood loss, and especially in the form of accidental haemorrhage associated with utero-placental apoplexy, the results cannot be expected to be so good.as in cases of placenta praevia and post-partum haemorrhage. Similarly, in cases of obstetric shock with no apparent bleeding it is difficult to know if blood transfusion is advisable; theoretically intravenous saline solution should be sufficient. In practice relatively few cases of obstetric shock occur apart from associated blood loss; hence transfusion is usually indicated.

\section{Incomplete Abortion}

Many cases of aborticn are admitted after great blood loss outside the hospital. The patients are still bleeding and show every likelihood of continuing to do so until the uterus has been emptied. In these cases a blood transfusion should be given and be followed immediately by emptying of the uterus. The latter procedure without preliminary transfusion is often fatal. In the past, since death so often occurred in the course of anaesthesia given to permit curetting, these fatalities were classified as due to anaesthesia, whereas the true cause was haemorrhage and shock.

\section{Dangers}

The main dangers are incompatibility, haemolysis, and acute dilatation of the heart.

Incompatible blood transfusion results in respiratory distress, cyanosis, mental excitement, and coma ; death occurs usually within two hours. Under hospital conditions it can be avoided by strict adherence to the rule of simultaneous compatibility tests by two different persons as indicated in this article under the heading "Methods." If any suspicion of incompatibility arises during the transfusion the procedure must be stopped at once.

Haemolysis will occur if the bloods are incompatible, or it may result from over-heating of the blood in the container. The duty of maintaining the blood in the container at body temperature is usually delegated to an assistant. If the assistant allows this blood to get more than a few degrees above the body temperature at any time after collection haemolysis may occur in the recipient's body, with the formation of haemoglobin casts in the renal tubules, and death from anuria six to twelve days later. If haemolysis is suspected large doses of alkali should be administered to prevent the formation of haemoglobin casts (Baker and Dodds, 1925).

Acute dilatation of the heart can be avoided by running the blood in at a proper rate. This complication should be specially watched for in cases with anaemia of some days' standing, in which fatty degeneration of the myocardium may be present. If acute dilatation of the heart occurs venesection must be performed at once for its relief.

The number of blood transfusions given in the Glasgow Royal Maternity and Women's Hospital were eleven in 1932, twelve in 1933, forty-three in 1934, 105 in 1935, and 165 in 1936. The average number of deaths from haemorrhage in the hospital for the five years 1930 to 1934 inclusive was 21.4 per year. In spite of the increased number of transfusions in 1935 sixteen deaths occurred from haemorrhage. The reason for this is that although transfusion was at that time being largely used for haemorrhage cases the worst cases still proved fatal because there was no means of securing suitable donors quickly. Similarly in the first six months of 1936 eight patients died from haemorrhage.

The new transfusion service was started in the middle of June, 1936. In the second six months of 1936 only two patients died from haemorrhage. The two deaths from haemorrhage since June, 1936, were both in cases of rupture of the uterus, one of which was not diagnosed. In the other a blood transfusion was given, but at the operation the bleeding points were not secured adequately, 
and further unrecognized internal bleeding occurred. On analysis of the deaths from haemorrhage recently it would appear that of the previous twenty cases annually about fifteen could have been saved by transfusion, but about five each year would have died despite transfusion owing to shock or to continued bleeding.

\section{Further Requirements}

What is still required is a method of keeping blood in stock similar to the storage of saline solution in bottles for intravenous use. Patients have died within half an hour of admission. An intravenous saline injection can be started within ten minutes of admission, and blood transfusion could be started in the same time if blood were kept in storage. I have had no experience of the Russian method.

\section{Conclusion}

From the statistics of the hospital about one-fourth of the deaths are due to haemorrhage. Of these deaths from haemorrhage about three-fourths can be avoided by transfusion within one hour of adinission to hospital. This has required the organization of a blood transfusion service for this hospital. Blood transfusion services are organized in London and certain other cities, but they should be organized by every obstetrical hospital when a public service is not available. These facilities should be extended to patients too ill to be brought into hospital at once and also to private practice in the area. For the smooth working of such a service the concentration of maternity work in a large centre with a highly skilled staff is preferable.

My thanks are due to the entire medical staff of the hispital for their co-operation.

REFERENCES

Baird, D. (1936). Lancet. 1, 395.

Baker and Dodds (1930). Brit. J., exp. Path., 6, 247.

S. C. Davidson and S. L. Warren (N.Y. St. J. Med., February 15,1937, p. 387 ) review radiographs of twentyfour patients with infectious arthritis taken before and after treatment. The sole measures used were fever therapy alone or in combination with a hot bath routine. Before treatment each patient was physically examined, septic foci were removed, and an electrocardiogram and a radiograph of the chest were taken. No dietetic regulations were enforced with the exception of an additional daily intake of one quart of milk. The patient was placed in a cabinet with the radiation from five 200 -watt lamps directed at the trunk and limbs, the head remaining outside. The temperature was raised to $40.5^{\circ} \mathrm{C}$. and maintained for four hours. A hot bath routine was carried out throughout the cold months of the year. Of twenty-four patients thus treated three showed no clinical or radiological improvement; six were clinically improved, but there was no radiological evidence of definite change in the bony structures round the joint to support this improvement; thirteen showed clinical and radiological improvement. The authors analysed their cases and found that cases of infectious arthritis are resistant to fever therapy $(a)$ when the disease is in the acute stage of soft tissue proliferation, and $(b)$ when the cartilage is affected and ankylosis has developed. These are usually persons under 30 years of age. In the subacute or chronic stage of infectious arthritis with thinning of the cartilage and bone atrophy in persons between 30 and 50 years of age marked improvement resulted. Two cases with degenerative hypertrophic arthritis obtained complete relief from symptoms after fever therapy, though without any radiological evidence of diminution of bony spur formation.

\section{THE PREVENTION OF CONSTIPATION}

BY

\section{E. M. DIMOCK, M.D., B.Ch.}

The progress of medicine is towards prevention. Yet constipation, one of the commonest ailments of civilization, receives only remedies. The present attitude of the medical profession towards constipation is deplorable. Students at many hospitals are brought up in an atmosphere where the indiscriminate use of purgatives is condoned. Too often the dose and type of purgative are left to the caprice of the nursing staff, and bear no relation to the experience of the patient. Recently, however, Professor Witts (1937) has shown that the treatment of acute constipation by purgation is irrational, and his article should be read by every doctor and nurse. As a general practitioner I treat numerous patients suffering from habitual constipation; I claim that this is a preventable malady and that the continued use of purgatives is irrational.

\section{Why the Abuse of Purgatives Continues}

The general practitioner is apt to be discouraged by many obstacles which prevent the effective treatment of habitual constipation. First, he is seldom instructed adequately on this subject while at hospital. Secondly, the diagnosis of habitual constipation can only be made by the patient, who is often misleading. Thirdly, no single factor can be blamed as the cause of constipation, and effective treatment must take into account all the circumstances in each case. At least four factors are commonly encountered:

1. The ignoring of the bowel habit or its lack of development.

2. Lack of physical activity.

3. Abuse of purgatives.

4. Unsuitable diets or artificially purified foods.

It follows that the treatment of habitual constipation is chiefly instructional. In actual practice this is so difficult that few doctors will make the attempt. Many patients have sedentary occupations, while others cannot afford a suitable diet. Moreover, there is nothing more difficult than to inculcate a habit into patients who have no use for any advice that requires personal trouble or thought. The rational treatment which at present is accepted by the medical profession does not utilize diet to the fullest advantage and, even when applied correctly, often fails altogether or only partially succeeds. It is discouraging for the doctor to take the time and trouble necessary to instruct his patients when his advice is not likely to be followed or, when utilized, is apt to fail. For these reasons the everyday treatment of constipation is largely disowned by the medical profession and is considered as the responsibility of the individual sufferer or parent; and patient, parent, and physician are all more concerned with the relief of attacks of constipation than with the prevention of their occurrence.

Purgatives appear to offer a simple and effective solution for doctor and patient. Under the term "purgative" I include aperients, salines, and all laxatives that are not purely mechanical. Not one of these "medicines" is physiological in action. I firmly balieve that the frequent use of purgatives is responsible for numerous minor ailments, especially digestive disorders, headaches, and nervous irritability. 